\title{
Investigation of star clusters found in the 2MASS catalog
}

\section{Elena Glushkova, ${ }^{1}$ Ramakant Yadav $^{2}$ and Sergey Koposov ${ }^{3,4}$}

${ }^{1}$ Sternberg Astronomical Institute, Universitetsky pr. 13, Moscow 119992, Russia

email: elena@sai.msu.ru

${ }^{2}$ Aryabhatta Research Institute of Observational Sciences,

Manora Peak, Nainital 263129, India

email: rkant@aries.res.in

${ }^{3}$ Max Planck Institute for Astronomy, Königsstuhl 17, Heidelberg 69117, Germany

email: koposov@mpia-hd.mpg.de

${ }^{4}$ Institute of Astronomy, University of Cambridge, Madingley Road, Cambridge CB3 0HA, UK

\begin{abstract}
We have launched a program of $U B V R$ CCD photometric investigations of new clusters detected in the 2MASs catalog based on an automatic detection method, which uses a convolution with a density function. Three new clusters, Koposov 12, Koposov 53 and Koposov 77 were observed in $U B V I$ with the $104 \mathrm{~cm}$ Sampurnanand telescope located at ARIES, Manora Peak, India. Their ages, distances, color excesses and metallicities have been derived using photometric data.
\end{abstract}

Keywords. open clusters and associations: general, open clusters and associations: individual (Koposov 12, Koposov 53, Koposov 77)

The full poster (in pdf format) is available at http://www . astro.iag.usp.br/ iaus266/Posters/pGlushkova.pdf. 